\title{
Reliability and Sensitive analysis of the Tooth Contact strength Based on Response Surface and Monte Carlo Methods

\author{
Yanyan Shi ${ }^{*}, 2$, Rong Yang ${ }^{2}$, Xiangfeng Kong ${ }^{2}$, Yang Cui ${ }^{2}$, Hong Sun ${ }^{2}$ and \\ Jinhua $\mathrm{Li}^{2}$
}

1. The State Key Laboratory of Mechanical Transmissions, Chongqing University,400044, China

2. AVIC Shenyang Engine Design and Research Institute Key Laboratory for Power Transmission Technology of Aero-engine, Shenyang, 110015, China

yanming7802@163.com

\begin{abstract}
Keywords: Thermo elastic coupling; Contact strength; Monte Carlo; Reliability sensitivity.
Abstract: The thermo-elastic contact characteristics of standard spur gears with the ANSYS software's Indirect coupling analysis method and achieve the limit state function of contact strength of tooth surface with the response surface method. It also studies the probabilistic sensitivity of the limit state function to the random variable with the reliable and sensitive analysis method of Monte Carlo and to achieve the Sensitivity information of various random factors on the reliability of contact strength of tooth surface under thermo elastic coupling state. Research results show that in the reliability design process the randomness of the temperature of the oil gas mixture in the accessory casing can be neglected and the constant treatment. It can effectively improve the gear tooth contact strength reliability through Increase the contact stress of the tooth surface, Reduce gear torque, Choose the gear material with smaller linear expansion coefficient and lower oil supply temperature and reduce the discrete range of random variable at the same time.
\end{abstract}

\section{Introduction}

With the development of aviation accessory drive to high speed and heavy load. A large amount of friction heat is generated by the gears mesh and formed a larger temperature gradient on the gears. Uneven temperature field distribution will cause thermal deformation. And superposition with the elastic deformation of the gear meshing, So it changes the stress state and contact tooth contact. Temperature field and thermal deformation of gear tooth are influenced by gear speed, transmission power, lubricant used, cooling facilities and the actual operating conditions, and other random factors. Meanwhile, These random factors also determine the contact state and the elastic deformation of the tooth surface when gear mesh ${ }^{[1,2]}$. These random factors are the root cause of the change of the contact stress of the tooth surface and has a great influence on the reliability of contact strength of tooth surface. Therefore, it is important to study the sensitivity of various random factors to the reliability of the contact strength of the tooth surface in thermal condition ${ }^{[3]}$.

When the distributed parameter of random variable are determined, the influence of random variables on the structural limit state function can reflect the influence of the random variable on the structural reliability. So we can effectively simplify the reliability sensitivity analysis work of complex structures. According to the results of reliability sensitivity analysis, we can regard the low sensitivity of the random variables as constant ,Simplify the factors of reliability design, improve the efficiency of reliability design. It is also suggested that the design parameters should be modified to improve the reliability of the structure and how to modify the design parameters. In this paper, the indirect coupled analysis method of ANSYS software is used to study the thermo elastic contact of a standard spur gear. The limit state function of contact strength of tooth surface is obtained by response surface method. Monte Carlo reliability sensitivity analysis method is used to study the probability sensitivity of each random variable to the limit state function. We can obtain sensitivity information of the reliability of the contact strength of the tooth surface with various random factors in the thermo elastic coupling condition ${ }^{[4,5]}$. 


\section{Response surface-the reliability sensitivity analysis method of Monte Carlo}

If we use the simulation method of Monte Carlo to cycle call directly. The finite element model of the calculation of a large amount of work, especially low probability problems often require more simulation times. The influence of the random variable on the structural reliability can be reflected by influence of the random variable on the structural limit state function. In this paper, the limit state function is obtained by using response surface method and the Monte Carlo sample is generated. The reliability sensitivity analysis method of the structure is accomplished by Carlo Monte simulation of the limit state function. This method is called the response surface-the reliability sensitivity analysis method of Monte Carlo.

By determining the distribution function of the random input variables and determine the actual range of the random input variables; Respectively measure the variation ranges of the model results are brought by the random input variables. Therefore, There are two factors that directly affect the results of probabilistic sensitivity analysis: discrete range of gradient and random input variables $X$ express random input variable $Y$ express model results. If $X$ has a given discrete range, Then the $Y$ discrete range is larger. The slope of the line is greater. As shown in Figure 1 (a) (The results of different models are represented by $Y_{1}, Y_{2}$ in the graph);But when $\mathrm{X}$ has a large discrete range, $Y$ with the same slope may also have considerable variation, as shown in Figure 1 (b).

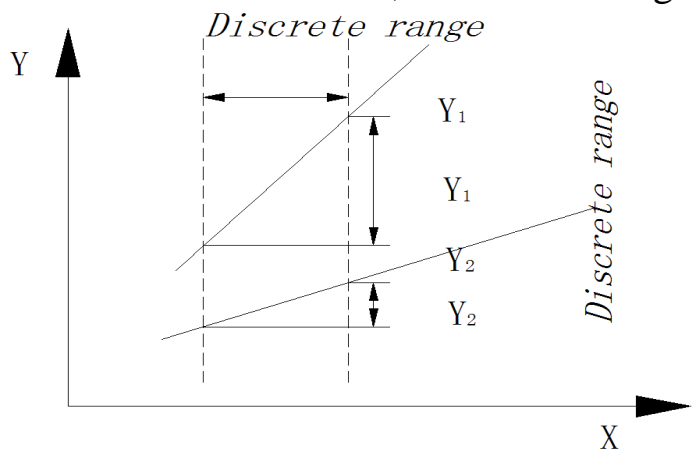

(a)

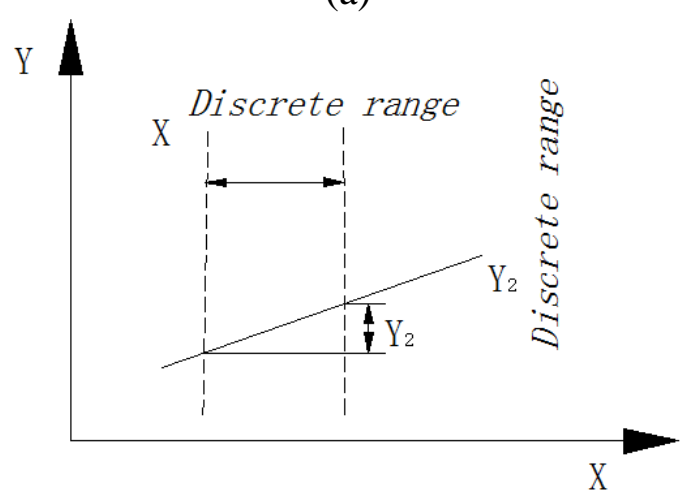

(b)

Fig. 1 Schematic diagram of probabilistic sensitivity analysis

Based on the response surface to establish the limit state function. Assumed contact stress of tooth surface is $p_{\text {lim }}$,By the failure theory of strength, The maximum contact stress $p_{\max }$ of the tooth surface is not allowed to exceed the allowable contact stress $p_{\lim }$, Therefore, the failure criterion for the contact strength of tooth surface is

$$
p_{\max } \geq p_{\text {lim }}
$$

Therefore, the limit state function for the reliability analysis of tooth surface contact strength is

$$
g(\boldsymbol{X})=p_{\lim }-p_{\text {max }}
$$

According to the response surface method, We can obtain the response surface function of the maximum contact stress of tooth surface and the limit state function of the response surface function. Which is shown in type (3), (4) respectively. 


$$
\begin{gathered}
p_{\max }=C_{0}+\sum_{i=1}^{N R} C_{i} X_{i}+\sum_{i=1}^{N R} \sum_{j=i}^{N R} C_{i j} X_{i} X_{j} \\
g(\boldsymbol{X})=p_{\lim }-p_{\max }=p_{\lim }-\left(C_{0}+\sum_{i=1}^{N R} C_{i} X_{i}+\sum_{i=1}^{N R} \sum_{j=i}^{N R} C_{i j} X_{i} X_{j}\right)
\end{gathered}
$$

$C_{0} 、 C_{i} 、 C_{i j}(i=1 \ldots N R ; j=i \ldots N R)$ is undetermined coefficient, A total of $N R+1+N R(N R+1) / 2$. $\mathrm{G}(\mathrm{X})$ the results represent the increase the reliability of the structure. $\mathrm{G}(\mathrm{X})$ the results represent the reduce the reliability of the structure. By studying the influence of each random variable and the degree of its influence. The sensitivity information of the random variables on the reliability of the structure can be obtained.

\section{Calculation example}

Known in the accessory drive system, A pair of engaged standard spur gears, Active tooth number $z_{1}$ is 24 . Driven tooth gear number is 38 , Gear modulus $\mathrm{m}$ is $3 \mathrm{~mm}$, Pressure angle is $25^{\circ}$, Breadth of tooth is $18 \mathrm{~mm}$, Gear material is $16 \mathrm{Cr} 3 \mathrm{NiWMoVNbE}$.Assembly temperature of accessory casing is $30^{\circ} \mathrm{C}$.Determine the working state, Linear expansion coefficient of the Gear material $l_{\alpha}$, Lubricating oil temperature $t_{\text {in }}$,Temperature of oil and gas mixture in accessory is $t_{\text {mix }}$, The active gear torque $\mathrm{M}$ and contact stress of tooth surface $p_{\text {lim }}$ are random variables, Assumed that the random variables are subject to normal distribution. The mean and standard deviation are shown in Table 1.

Table 1 mean and standard deviation of the random variables

\begin{tabular}{cccccc}
\hline Variables & $l_{\alpha}\left(10^{-6} /{ }^{\circ} \mathrm{C}\right)$ & $t_{\text {in }}\left({ }^{\circ} \mathrm{C}\right)$ & $T_{\max }\left({ }^{\circ} \mathrm{C}\right)$ & $M(\mathrm{~N} . \mathrm{m})$ & $P_{\lim }(\mathrm{MPa})$ \\
\hline Mean & 11.6 & 90 & 103.602 & 175.522 & 163550 \\
Standard deviation & 0.5 & 4 & 5.5 & 6 &
\end{tabular}

The boundary conditions of the finite element model for the finite element model of the meshing gears are first applied, Solving heat analysis model, Get the temperature field of the main and driven gear. Reconvert unit type, The thermal analysis element in the finite element model is converted to a structural analysis unit. The mechanical load is further applied to the finite element model. Meanwhile. Thermal load and mechanical load are applied to the finite element model of the meshing gear pair at the same time, as shown in Figure 2. Choose Newton-Raphson balanced iterative algorithm, Then solve the structural analysis model of the thermal load and mechanical load, The equivalent stress contours of the main and driven gears are shown in Figure 3.

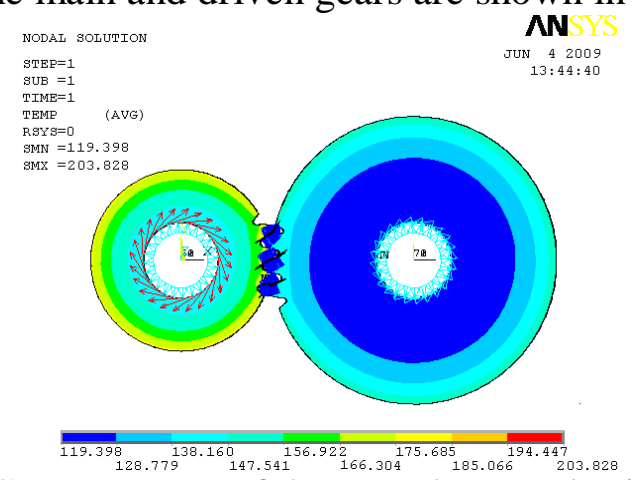

Figure 2 Contact stress of the gear thermo elastic coupling
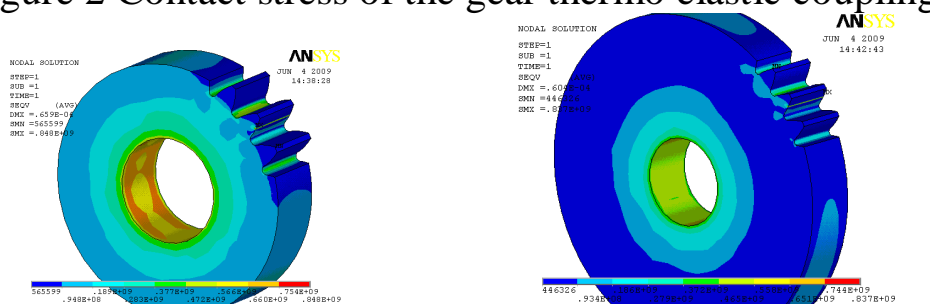

Figure 3. Equivalent stress cloud of the active gear and the driven gear

Probability level are $p_{1}=0.1 、 p_{2}=0.5 、 p_{3}=0.9$, Sample the sample points are generated according to the random variable Box-Behnken ${ }^{[6]}$ sampling method in Table 1, As shown in Table 2. 
Table 2 Box-Behnken sample points and response values

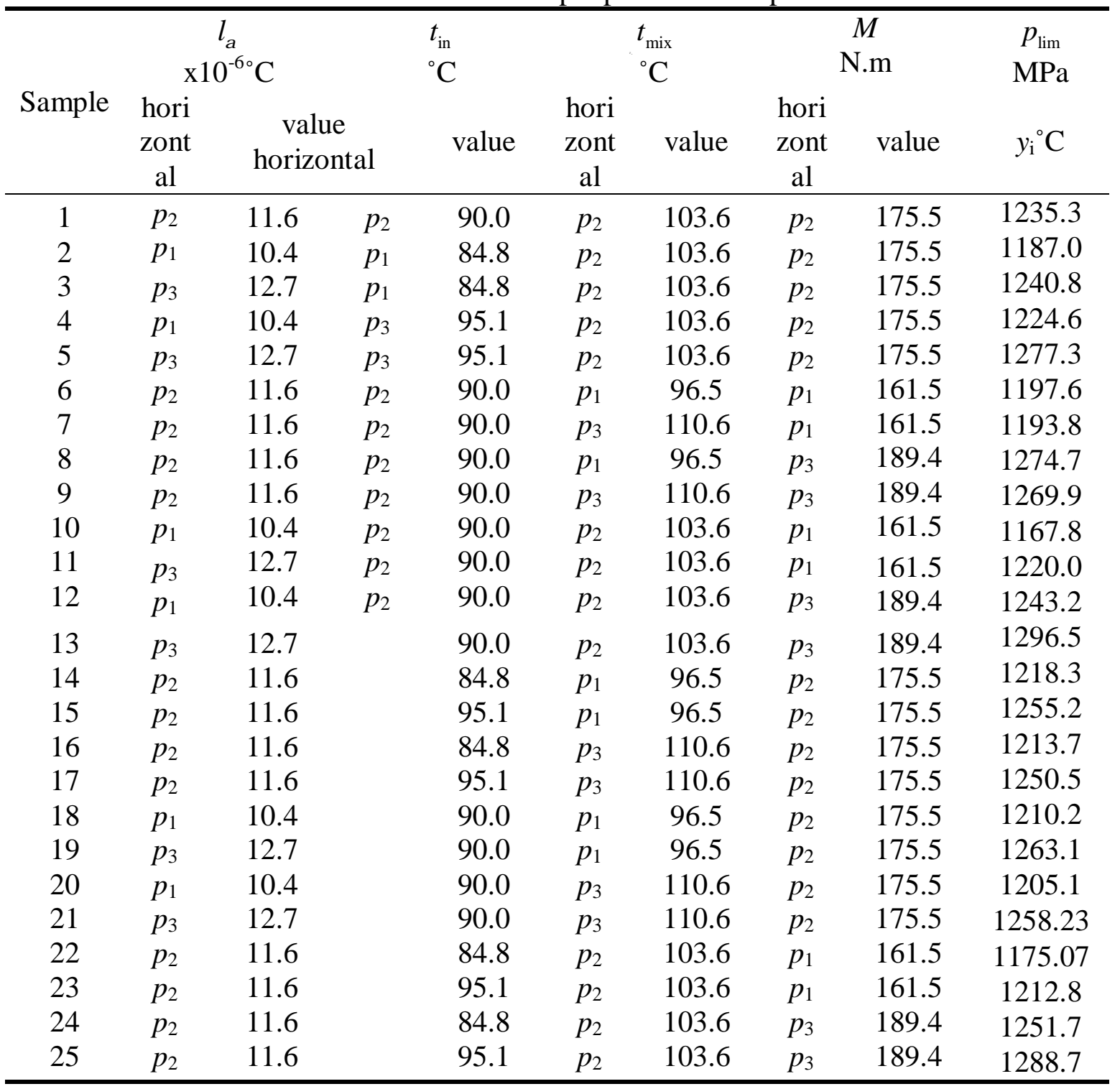

The maximum contact stress of the tooth surface is obtained by the finite element simulation of the sample points in 25 times, The last column in Table 2.The response surface function of the maximum contact stress of the tooth surface is obtained according to the data in Table 2.

$$
\begin{gathered}
p_{\max }=-58.962+133.5068 l_{\alpha}+14.0728 t_{\mathrm{in}}-2.2955 t_{\text {mix }}-8.7558 M-3.7814 l_{\alpha}^{2} \\
-0.0791 l_{\alpha} t_{\text {in }}+0.015 l_{\alpha} t_{\text {mix }}+0.007 l_{\alpha} M-0.0519 t_{\mathrm{in}}^{2}-0.0009 t_{\mathrm{in}} t_{\text {mix }} \\
-0.0007 t_{\mathrm{in}} M+0.0095 t_{\text {mix }}^{2}-0.0006 t_{\text {mix }} M+0.0377 M^{2}
\end{gathered}
$$

Therefore, The limit state function $g(\boldsymbol{X})$ of the contact strength of the tooth surface is obtained by using the response surface method.

$$
\begin{aligned}
& g(\boldsymbol{X})=p_{\text {lim }}-p_{\text {max }}=p_{\text {lim }}+58.962-133.5068 l_{\alpha}-14.0728 t_{\text {in }}+2.2955 t_{\text {mix }} \\
& +8.7558 M+3.7814 l_{\alpha}^{2}+0.0791 l_{\alpha} t_{\text {in }}-0.015 l_{\alpha} t_{\text {mix }}-0.007 l_{\alpha} M+0.0519 t_{\text {in }}^{2} \\
& +0.0009 t_{\text {in }} t_{\text {mix }}+0.0007 t_{\text {in }} M-0.0095 t_{\text {mix }}^{2}+0.0006 t_{\text {mix }} M-0.0377 M^{2}
\end{aligned}
$$

According to the random variable and its distribution parameters in Table 1, The limit state function is simulated by Carlo Monte for 1000 times, 1000 samples of the function value $\mathrm{Y}$ are generated,As is shown in figure 4. 


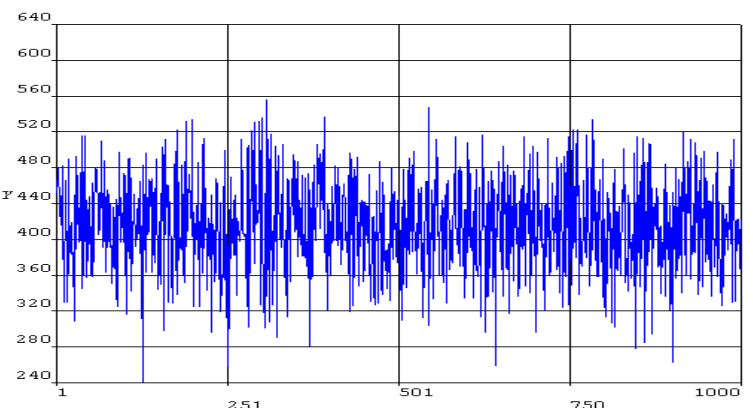

Figure 4 Sample history of Figure 4Y

\section{Conclusion}

The sensitivity of the reliability of the random variables to the contact strength of the tooth surface under the thermo elastic coupling state is studied by using the method of response surface and Carlo Monte reliability sensitivity analysis. The reliability sensitivity analysis of complex structures can be simplified by this method.

In the reliability design process, The randomness of the temperature of the oil gas mixture in the accessory casing can be neglected and the constant treatment; By improving the contact stress of the tooth surface, reduce gear torque, select the gear material with smaller linear expansion coefficient and lower oil supply temperature, At the same time reduce the discrete range of the random variables, Can effectively improve the contact strength reliability of the tooth surface.

\section{References}

[1] Runfang Li ,Yusheng Li.Coupled thermo elastic contact finite element method and its application in gear transmission [J],Journal of Chongqing University,1993, 16(1): 96-101.

Reference to a book:

[2] Chengli Liu,Zhenyu Lv.An improved response surface method for the probabilistic analysis of structural systems with implicit limit state equation [J],Mechanical strength,2006, 28(3): 358-362

[3] Bjerager P, Krenk S. Parametric sensitivity in first order reliability theory [J], Journal of Engineering Mechanics, ASCE, 1989, 115(7): 1577-1582.

[4] Wei Duan,Zhangqi Wang.Probability strength design and sensitivity analysis of steam turbine blade based on response surface method [J],Chinese Journal of Electrical Engineering,2007, 27(5): 99-104.

[5] Mary J, Lindstrom and Douglas M. Bates. Newton-Raphson and EM algorithms for linear mixed-effects models for repeated-measures data [J], Journal of the American Statistical Association, 1988, 83(404): 1014-1022.

[6] Box G E P, Behnken D W. Some new three level designs for the study of quantitative variables [J], Technometrics, 1960, 2(4): 455-476. 